\title{
A New and Efficient 2D Collision Detection Method Based on Contact Theory
}

\author{
Xiaolong CHENG, Jun XIAO ${ }^{a}$, Ying WANG, Qinghai MIAO, Jian XUE \\ University of the Chinese Academy of Sciences \\ No.19A Yuquan Road, Beijing, 100049, China \\ aemail: xiaojun@ucas.ac.cn
}

Keywords: Contact Collision; Contact Theory; Entrance Block; Contact Cover

\begin{abstract}
Collision detection is a fundamental problem in mechanical, robotic, structural, and geotechnical engineering. A new algorithm base on Contact Theory to solve accurate contact problem is proposed in this paper. In this method, two polygons (A and B) and a reference point are defined, where the reference point has parallel movement to polygon $\mathrm{A}$. Then we calculate contact covers, build boundary of Entrance Block, and the contact problem between two general polygons is reduced to contact between a reference point and the boundary of Entrance Block. i.e., we can get distance between two polygons if they are separated, get contact points if they contact, and get shortest exiting path along with contact points if they are intersected. Experiments and analysis show that the proposed method can compute the collision detection problem accurately and reduce the complexity of contact problem obviously.
\end{abstract}

\section{Introduction}

Collision detection is a basic but important problem in computer graphics, and it is an unavoidable problem for analysis of almost all moving geometry objects. Polygons with different positions have different contact points, and there may be infinite contact points when polygons are in certain positions. As a result [1], this problem has drawn much attention of scholars from different fields in the past decades. Several strategies have been developed in order to deal with the problem [2], such as the common plane [3], the single surface algorithm [4], hierarchy territory algorithm [5], the "master-slave" approach [6], the "material depth" approach [7] and the polygon-particle algorithm [8]. In addition, there are also some strategies for rigid bodies, e.g. the "Optimization Based Animation (OBA)" approach [9] for computing graphics purposes [10, 11] and the "Non Smooth Contact Dynamic (NSCD)" formulation [12].

Contact Theory [1] is the first theory that presents and proves contact problem in mathematics. It is different from all existing contact algorithms, since it provides a general way to solve contact problem. In Contact Theory, a new concept "Entrance Block" is introduced. By means of Entrance Block, the contact problem between two general polygons reduces to contact between a reference point and the Entrance Block, which simplifies the complexity of the contact problem. In this paper, we just give a 2D contact algorithm, but it is still important that 2D result is conservative and can be accepted easily in same cases (e.g., many worldwide major engineering projects are analyzed by 2D-DDA [13]).

We give basic concepts of Contact Theory in section 2, algorithm in section 3, and two examples in section 4, and conclusion in section 5 .

\section{Some Base Concepts of Contact Theory}

In this section, we define the geometry data structure, and give brief explanation of some basic concepts and simple application of Contact Theory [1]. All geometry data structure obey right-hand coordinate, as shown in figure 1: line segment $A$ (figure $1 . a)$ is represented by two points $\left(a_{0}\right.$ and $\left.a_{1}\right)$ with direction $\mathrm{a}_{1}-\mathrm{a}_{0}$; angle $\mathrm{A}$ (figure 1.b) is represented by two line segments (i.e. $\mathrm{e}_{1}$ and $\mathrm{e}_{2}$ ) with the sector rotates from $\mathrm{e}_{1}$ to $\mathrm{e}_{2}$; polygon A (figure 1.c) is represented by its points (i.e., $\mathrm{a}_{0} \ldots \mathrm{a}_{4}$ ), its 
edges (i.e., $\mathrm{e}_{0} \ldots \mathrm{e}_{4}$ ), and its area.

The basic concepts of Contact Theory are relations of two polygons, reference point, contact cover and Entrance Block. The relations of two polygons are separated, contacted and intersected. Moreover, the contact relation is what we concentrate on.

The reference point is a key concept in Contact Theory. In Contact Theory, two polygons are named as polygon $\mathrm{A}$ and polygon $\mathrm{B}$, and the reference point has parallel movement to polygon A. Then we can easily locate polygon A by the reference point. In addition, the reference point is used to calculate Entrance Block and detect current contact.

The contact cover contains contact information of two geometry objects, and can overlap with each other. Contact covers are calculated by the core formula expressed as formula (1), where A and $\mathrm{B}$ represent polygons, while p represents reference point.

$$
E(A, B)=B-A+p
$$

For example, giving point "a” and line segments B (figure 2), their contact cover is a line segment (from $p$ to $p$ ') with B fixed. We can determine the certain position of point "a” by every point on the contact cover. For overlapping (figure 3), we can get two contact covers if reference point is on the bottom contact cover $E\left(a_{i} a_{i+1}, b_{j} b_{j+1}\right)$.

The Entrance Block is the key concept of Contact Theory. It consists of contact covers, and contains all contact information of A and B with B fixed. We can understand Entrance Block in a simpler way that a geometry object composed by the positions of reference point when two polygons contact or intersect.

To detect current contact, we only need the boundary of Entrance Block. Therefore, before detecting contact, we need to build boundary of 2D Entrance Block. The boundary is a contact cover system, which consists of contact edges or angles (figure 4). Taking advantages of Contact Theory, we detect current contact easily that what we do is to select the contact edges with shortest distance to reference point. Thus, contact computation is simplified with reference point and Entrance Block: for separated case (figure 5.a), we get the shortest distance between the reference point and the Entrance Block, which is also the shortest distance between two polygons; for contact case (figure 5.b), we get contact points and contact locations of two polygons; for intersected case (figure 5.c), we get the contact points and shortest exiting path, which is the line between reference point and its projective point on the Entrance Block.

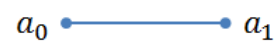

a. Line Segment

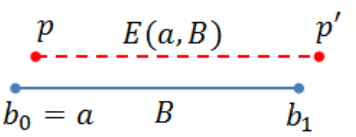

Figure 2 contact cover of point and line segment

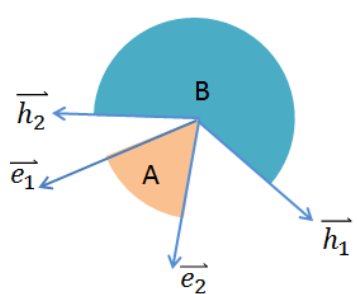

b. Convex and Concave Angles

Figure 1 Geometry Shapes

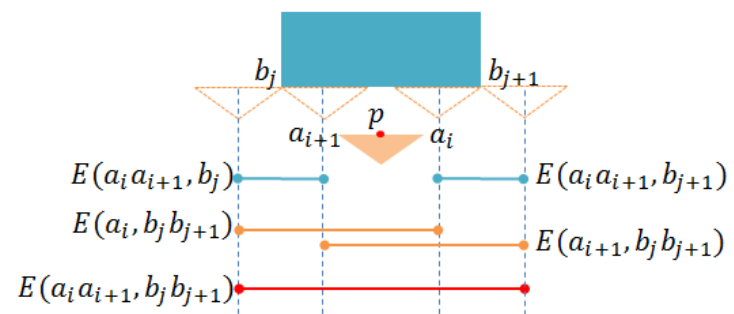

Figure 3 Overlapped Contact Covers

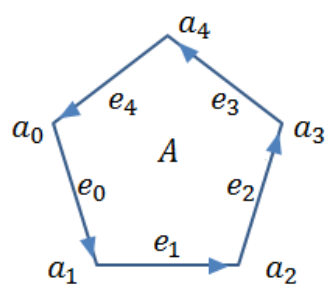

c. Polygon

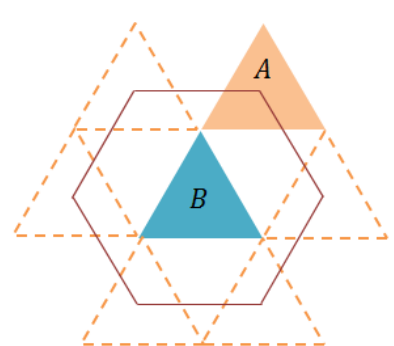

Figure 4 Entrance Polygon of two Triangles 


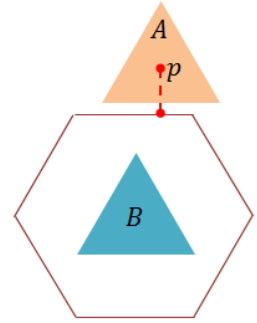

a. Separated Condition

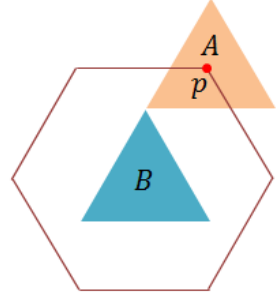

b. Contacted Condition

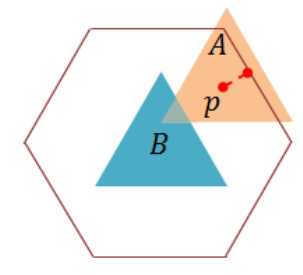

c. Intersected Condition

Figure 5 Judging Relations of Polygons with Reference Point and Entrance Block

\section{D Algorithm to Detect Contact with Contact Theory}

Entrance Block is complex, but we only need its boundary to detect current contact. There are 3 phases to detect the current contact covers: 1 ) define reference point and two polygons (A and B); 2) calculate contact covers and compose boundary of Entrance Block; 3) detect current contact covers with reference point. We will give the phases in detail next.

(1)Algorithms for Calculating Contact Covers

For 2D contact algorithm, we consider angle-edge and angle-angle contact. Moreover, the result is both contact edges. Contact edges of Angle-Edge and Edge-Angle are calculated by formula 2 (figure 6) and formula 3 (figure 7), while contact edges of Angle-Angle is calculated by formula 4 (figure 8).

$$
E(a, B)=\left\{b_{1}+t *\left(b_{2}-b_{1}\right)-a+p: t \in[0,1]\right\}
$$

Where vertex of angle $\mathrm{A}$ is point $\mathrm{a}$, points of edge $\mathrm{B}$ are $\mathrm{b}_{1}$ and $\mathrm{b}_{2}$, and $\mathrm{p}$ is the reference point.

$$
E(A, b)=\left\{b-\left(a_{1}+t *\left(a_{2}-a_{1}\right)\right)+p: t \in[0,1]\right\}
$$

Where points of edge $A$ are $a_{1}$ and $a_{2}$, vertex of angle $B$ is point $b$, and $p$ is the reference point.

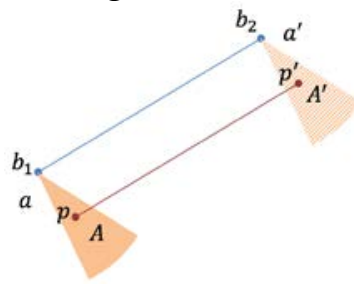

Figure 6 Angle-Edge contact

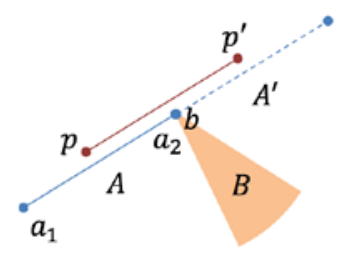

Figure 7 Edge-Angle contact

$$
E(A, B)=\left\{\begin{array}{ll}
E\left(A, h_{1}\right): & e_{1} \times h_{1} \text { u } \\
E\left(e_{1}, B\right): & \text { others }(0,0,1)
\end{array}\right\} \cup\left\{\begin{array}{ll}
E\left(e_{2}, B\right): & \begin{array}{l}
\mathbf{u} e_{2} \times h_{2} \uparrow \uparrow(0,0,1) \\
E\left(A, h_{2}\right):
\end{array} \text { others }
\end{array}\right\}
$$
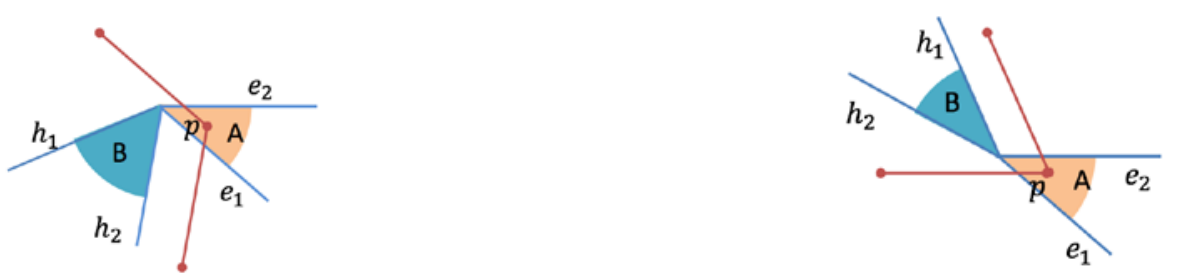

a. Angle-Angle Contact b. Angle-Angle Contact

Figure 8 Contact covers of Angle-Angle Contact

(2)Calculate Boundary of Entrance Block

With the formulas (2), (3) and (4), we give the processes to calculate boundary of Entrance Block here. According to the above description, we only need to calculate valid (i.e., for angle-angle contact, angles must not intersect if their vertices move to one point; for angle-edge contact, they must not intersect if angle's vertex moves onto edge) contact edges to solve 2D contact problems. To judge intersection, there are two institute ways: using vector calculation or using angle calculation. We use judging algorithms by vector calculation, which is given as formula (5) and (6), as shown in figure 9 and 10 . 
With formulas (2)-(6), we now give the processes (figure 11) to build the boundary of Entrance Block. There are three steps: 1) select disjoint convex angles from polygon A and B, and calculate the first angle-edge contact cover; 2) select next angle along the edge by right-hand system, and calculate next contact edge; 3) repeat step 2 until we get the starting two angles. For algorithm in this paper, we do not consider straight angles for that omitting straight angles will reduce computing and does not change shape of Entrance Block.

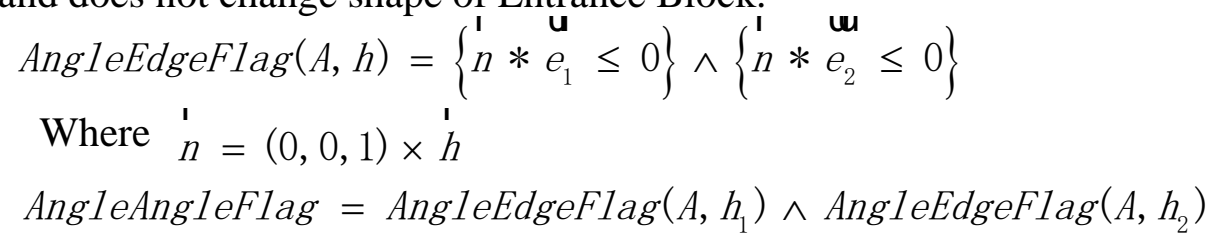

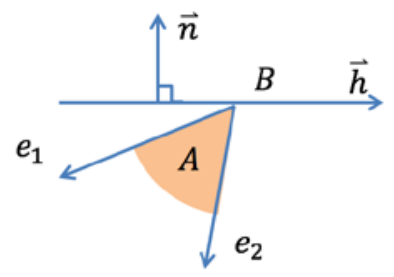

Figure 9. Angle-Edge Intersection Judgment

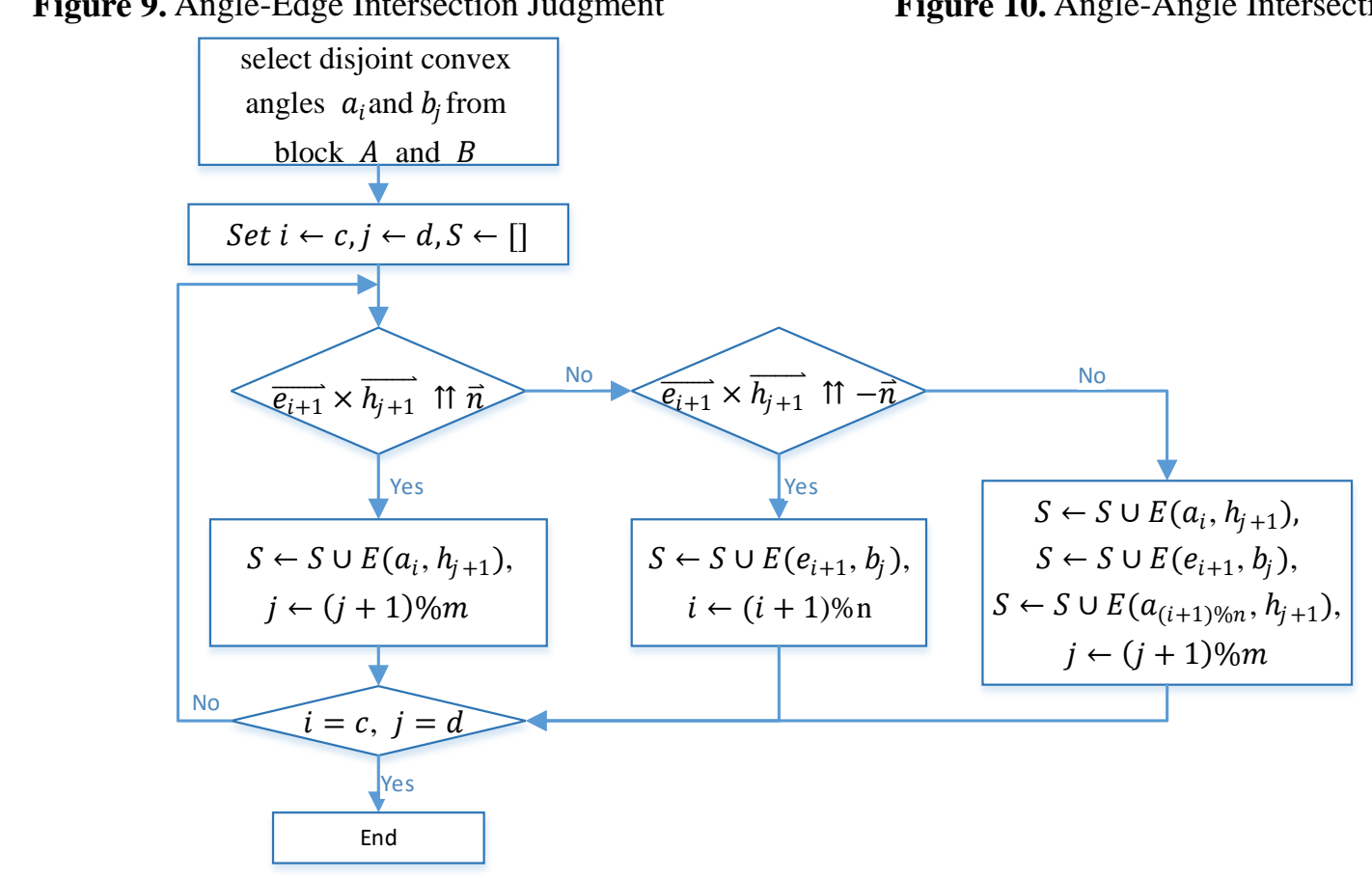

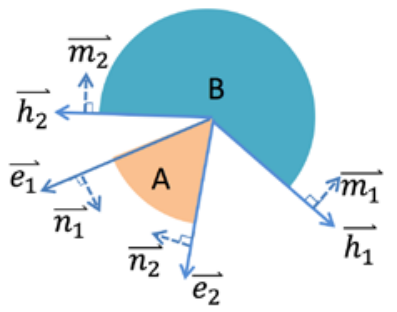

Figure 11. Flow chart of Contact Algorithm for Convex Polygons

(3)Detect current contact

After Entrance Block built, we use the reference point and Entrance Block to detect current contact. "current contact" means that the contact is happening, as shown in figure 12 and 13 that two contact edges works at the same time. To detect current contact, we calculate distance between the reference point and contact edges one by one according to formula (8), and collect contact edges with shortest distance by formula (9). There are two things left to be done: (1) we must check the collected contact edges to get if any two contact edges can compose an angle-angle contact cover; (2) recalculate and reselect contact edges for the ones that the edge of Angle-Edge contact cover contains straight angles.

$$
\operatorname{dist}(e, p)=V *\left(p-e_{1}\right)
$$

Where $v=$ normalize $(e \times(0,0,1))$, and $\mathrm{e}_{1}$ is the start point of contact edge e.

$$
R=\left\{e: \min _{e \in S}(\operatorname{fabs}(\operatorname{dist}(e, p)), \text { for all } \operatorname{dist}(e, p) \leq 0\}\right.
$$


Where S contains all calculated contact covers.

\section{Analysis and Experiments}

In this section, we analyze the algorithm complexity and give two examples to explain the process of getting current contacts with the proposed algorithm.

Usually, accurate collision detection algorithms need to check each Angle-Edge and Angle-Angle cases, which has a complexity of " $2 * O(m * n)+O(m * n)=O(m * n)$ " if polygon A and $\mathrm{B}$ have $n$ and $m$ points separately. But in the same condition, the complexity of the proposed algorithm is just " $O(m+n)+O(m+n)=O(m+n)$ ".

Now we give two examples. The first one shows how to detect contact for convex polygons without parallel edges (figure 12), while the second one uses convex polygons with 10 points (figure 13). For the two examples, we define polygon A with blue color, polygon B with yellow color, and reference point being the center of polygon A. The contact covers of Angle-Edge (angle of A to edge of B) are in red color, while contact covers of Edge-Angle (edge of A to angle of B) are in blue color.

(1)Experiment for Convex Polygons without Parallel Edges

The case of convex polygons without parallel edges is the simplest example. The Entrance Block is shown in figure 12(a); contact covers of Angle-Edge are shown in figure 12(b); contact covers of Edge-Angle are shown in figure 12(c). If we set polygon $\mathrm{A}$ at position of the hollow triangle in figure 12(d), we can get an Edge-Angle contact cover with reference point and Entrance Block.

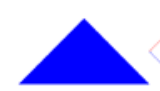

a. Entrance Block

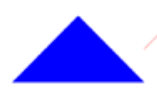

b. Contact Covers of Angle-Edge

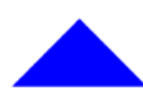

c. Contact Covers of Edge-Angle

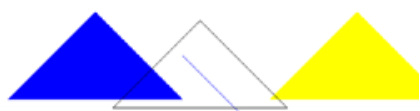

d. Selecting working Contact Covers

Figure 12. convex polygons without parallel edges

(2)Experiment for Convex Polygons with 10 edges

In real computing, it is often to meet the case of calculating contact with circles. The algorithms calculate the case for circles as well if we discretize circle into line segments. In order to facilitate the demonstration, we just discretize circle into 10 line segments. The Entrance Block is shown in figure 13(a); contact covers of Angle-Edge are shown in figure 13(b); contact covers of Edge-Angle are shown in figure 13(c). If we set polygon A at position of the hollow circle in figure 13(d), we can get two Angle-Edge contact covers and one Edge-Angle contact cover in two directions. Moreover, we need to determine which exiting path along with the corresponding contact covers is to be used next. It should be mentioned that this is an Angle-Angle contact according to the contact edges.

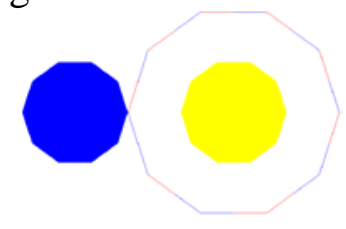

a. Entrance Block

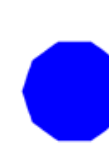

b. Contact Covers of Angle-Edge

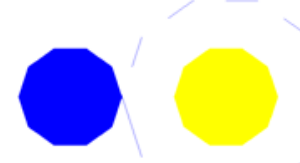

c. Contact Covers of Edge-Angle

Figure 13. convex polygons with parallel edges

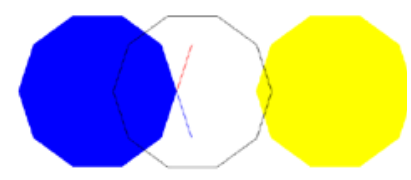

d. Selecting working Contact Covers

\section{Conclusion}

Contact Theory has opened a new filed for solving contact problem. The algorithms proposed in this paper realize it for 2D convex polygons, and provide a fast and robust algorithm to detect contact. In further work, we will work to calculate the cases of convex polygons and start to design algorithms for 3D case. Moreover, as we see in this paper, boundary of Entrance Block contains all contact information, but we only need a small part of boundary of Entrance Block to find the needed contact covers. Therefore, we will try to find a way to calculate smallest partial boundary of 
Entrance Block that contains needed contact information in further work.

\section{Acknowledgement}

Thanks a lot for Dr. Shi detailed instructions and supervision. This work is supported by the National Natural Science Foundation of China (No.61471338), President Fund of UCAS, Youth Innovation Promotion Association CAS (2015361).

\section{Reference}

[1] G.H. Shi. Contact theory. Article Special Issue On Rock Fractures And Discontinuities: Modeling And Analysis Science China Technological Sciences. 2015, 58(9): 1450-1496

[2] W. P. Computational Contact Mechanics. J. Wiley \& Sons, New York, 2002.

[3] Cundall P A. Formulation of a three-dimensional distinct element model-Part I: a scheme to detect and represent contacts in a system composed of many polyhedral blocks. Int J Rock Mech Min, 1988, 25: 107-116

[4] Benson D J, Hallquist J O. A single surface contact algorithm for the post-buckling analysis of shell structures. Comput Method Appl M, 1990, 78: 141-163

[5] Zhong Z H, Nilsson L. A contact searching algorithm for general contact problems. Comput Struct, 1989, 33: 197-209

[6] Jeleni' c G, Crisfield M A. Non-linear master-slave relationships for joints in 3D beams with large rotations. Comp Meth Appl Mech Engng, 1996, 135: 211 - 228

[7] G. Hirota, F. S., and S. A. An improved finite-element contact model for anatomical simulations. The Visual Computer, 19(5):291-309, 2003.

[8] Li S H, Zhao M H, Wang Y N, et al. A new numerical method for DEM-block and particle model. Int J Rock Mech Min, 2004, 41

[9] V. J. Milenkovic and H. Schmidl. Optimization-based animation. In SIGGRAPH '01: Proceedings of the 28th annual conference on Computer graphics and inter active te chniques, pages 37-46, New York, NY, USA, 2001. ACM Press.

[10] D. Baraff. Analytical methods for dynamic simulation of non-penetrating rigid bodies. In SIGGRAPH '89: Proceedings of the 16th annual conference on Computer graphics and interactive techniques, pp 223-232, New York, NY, USA,1989. ACM Press.

[11] D. Baraff and A. Witkin. Large steps in cloth simulation. In SIGGRAPH '98: Proceedings of the 25th annual conference on Computer graphics and interactive techniques, pages 43-54, New York, NY, USA, 1998. ACM Press.

[12] M. Jean, V. Acary, and Y. Monerie. Non-smooth contact dynamics approach of cohesive materials. R. Soc. Lond. Philos. Tr ans. Ser. A Math. Phys. Eng. Sci., 359(1789):2497-2518, 2001. Non-smooth mechanics.

[13] Shi, G.H. Discontinuous deformation analysis - A new numerical model for the statics and dynamics of block systems. Ph.D. thesis. 1988. Univ. of California, Berkeley, Calif. 